\title{
Angiosarcoma and anaplastic carcinoma of the thyroid are two distinct entities: a morphologic, immunohistochemical, and genetic study
}

\author{
Elisabetta Kuhn ${ }^{1} \cdot$ Moira Ragazzi ${ }^{1} \cdot$ Alessia Ciarrocchi ${ }^{2} \cdot$ Federica Torricelli $^{2} \cdot$ Dario de Biase $\mathbb{D}^{3} \cdot$ Eleonora Zanetti ${ }^{1}$. \\ Alessandra Bisagni ${ }^{1}$ - Stefania Corrado ${ }^{4}$ - Silvia Uccella ${ }^{5}$. Stefano La Rosa ${ }^{6}$ Massimo Bongiovanni $\mathbb{C}^{6}$. \\ Simona Losito ${ }^{7} \cdot$ Simonetta Piana $\mathbb{1}^{1}$
}

Received: 2 November 2018 / Revised: 27 November 2018 / Accepted: 27 November 2018 / Published online: 5 February 2019

(c) United States \& Canadian Academy of Pathology 2019

\begin{abstract}
Angiosarcoma and anaplastic carcinoma are the most lethal neoplasms of the thyroid worldwide and share some similarities, which have led to a longstanding controversy on their etiopathological relationship. Thyroid angiosarcomas are characterized by vessel formation and an immunophenotype common to endothelial cells, while anaplastic carcinomas are partially or wholly composed of mesenchymal-like cells that have lost the morphologic and functional features of normal thyroid follicular cells. To investigate whether angiosarcomas represent the endothelial extreme of the differentiation spectrum of carcinomas or they are bona fide vascular neoplasms, we studied the clinico-morphologic and genetic characteristics of a series of 10 angiosarcomas and 22 anaplastic carcinomas. Immunohistochemically, among the endothelial markers, CD31 and ERG were the most consistently expressed in angiosarcomas. Among the markers of thyroid origin, PAX8 was the most reliable in anaplastic carcinomas, while TTF-1 reactivity was found in only $5 \%$ of anaplastic carcinomas and thyroglobulin was always negative. Pankeratin reacted with most angiosarcomas and anaplastic carcinomas and is therefore not useful in the differential diagnosis. Interestingly a mutated pattern of p53 immunostaining prompted a diagnosis of anaplastic carcinoma. To compare the genetic profile, we used the NGS approach to sequence hotspot regions within a panel of 57 genes. As a result, only a few mutations were found in angiosarcomas and all of them were single events (no TP53 or TERT mutation). On the other hand, anaplastic carcinomas were characterized by a higher number of mutations, and TP53 and TERT promoter mutations were the most frequent genetic alterations. The lack in angiosarcomas of the common mutations identified in anaplastic carcinomas supports a different genetic origin and strongly suggests that, in spite of a shared sarcomatous morphology and a similar clinical aggressiveness, angiosarcomas and anaplastic carcinomas rely on a completely different set of genetic alterations during their evolution.
\end{abstract}

These authors contributed equally: Elisabetta Kuhn, Moira Ragazzi

Simonetta Piana

simonetta.piana@ausl.re.it

1 Pathology Unit, Arcispedale Santa Maria Nuova, Azienda USLIRCCS, Reggio Emilia, Italy

2 Laboratory of Translational Research, Arcispedale Santa Maria Nuova, Azienda USL-IRCCS, Reggio Emilia, Italy

3 Department of Pharmacy and Biotechnology (Dipartimento di Farmacia e Biotecnologie) - Molecular Pathology Unit, Azienda

\section{Introduction}

Angiosarcoma and anaplastic carcinoma are the most lethal neoplasms of the thyroid worldwide. Together they account for less than $5 \%$ of clinically malignant thyroid neoplasms; however, they are responsible for most of the fatal

USL di Bologna, University of Bologna, Bologna, Italy

4 Department of Anatomic Pathology, Azienda OspedalieroUniversitaria, Modena, Italy

5 Department of Medicine and Surgery, University of Insumbria, Varese, Italy

6 Service of Clinical Pathology, Lausanne University Hospital, Institute of Pathology, Lausanne, Switzerland

7 National Cancer Institute G. Pascale, Naples, Italy 
outcomes. Interestingly, despite their rarity, their incidence shows a geographic variability, being higher in Europe than in the USA and seems associated with a dietary iodine deficiency, in particular on early literature [1].

Thyroid angiosarcomas are characterized by vessel formation and an immunophenotype common to endothelial cells, including CD31, CD34, and ERG positivity. Many angiosarcomas in the thyroid are epithelioid, as they are characterized by large epithelioid cells, with vescicolous nuclei, prominent nucleoli, and abundant eosinophilic cytoplasm; moreover, they mostly express immunohistochemical positivity for cytokeratin. On the other hand, anaplastic carcinomas are partially or wholly composed of mesenchymal-like cells that have lost the morphologic and functional features of normal thyroid follicular cells, including the ability to uptake iodine.

The morphologic and immunophenotypic similarities between angiosarcomas and anaplastic carcinomas led to a longstanding controversy on the etiopathological relationship between these entities that held the stage in the 80-90 s and still remains unresolved. This debate mainly counterpoised American to European pathologists. The American pathologists believed that carcinomas of the thyroid could rarely exhibit endothelial features; therefore, angiosarcomas would represent a variant of anaplastic carcinomas, namely, the endothelial extreme of their differentiation spectrum [2-5]. In contrast, European pathologists claimed that angiosarcoma is a bona fide pure vascular neoplasm and, accordingly, a true entity on its own [6-11].

To address this issue, some immunohistochemical studies provided evidence of immunophenotypic differences between angiosarcomas and anaplastic carcinomas [12-15]. In addition, only one molecular study stated that angiosarcomas and anaplastic carcinomas are unrelated malignant tumors, based on the differential expression of thyroglobulin mRNA [16]. In 2017, the revised WHO classification of tumors of endocrine organs defined thyroid angiosarcoma as "a primary thyroid tumor showing evidence of endothelial cell differentiation"; however, it conceded that "there seems to be no concrete means of resolving the conceptual issue" of its pathogenesis [1].

In the past, the distinction between thyroid angiosarcomas and anaplastic carcinomas could have been considered a mere academic, sterile debate since the limited therapeutic possibilities could not allow a differential therapeutic approach [17]. Currently, the implementation of genomic profiling to explore molecular diversity or similarity between angiosarcomas and anaplastic carcinomas, together with the large availability of target drugs, holds profound implications for the improvement of tailored effective treatment strategies that would improve the prognosis of these ominous diseases [18].
Thus, we undertook the current study to investigate the clinicopathologic features, immunophenotypes, and molecular characteristics of a series of angiosarcomas compared to anaplastic carcinomas in order to systematically assess their differences and similarities and clarify their pathogenetic relationship.

\section{Material and methods}

\section{Case selection}

Pathology reports from the electronic archives of our Institutions were searched for cases of angiosarcomas and anaplastic carcinomas of the thyroid from 1978 to 2016. Only surgically resected tumor cases were included in this study; therefore, patients with an angiosarcoma or an anaplastic carcinoma diagnosis based on cytological techniques or small biopsies were excluded.

Three pathologists (EK, MR, and SP), experts in thyroid pathology, reviewed all the available slides with the achievement of a consensus evaluation, according to the current WHO classification of tumors of endocrine organs [1]. The study cohort was thus composed of 10 angiosarcomas and 22 anaplastic carcinomas patients.

Clinicopathological information (including patient sex, age, surgical procedures, postsurgical therapies, and followup) was obtained for each patient.

This research was conducted upon the approval of the Institutional Review Board of the Arcispedale Santa Maria Nuova - IRCCS, Reggio Emilia, Italy (protocol no. 71141-2017).

\section{Morphologic and immunohistochemical analysis}

At least 4 hematoxylin and eosin slides per case, median 6 , were available for review.

The most representative paraffin block per case was selected for both immunohistochemical characterization and next-generation sequencing analysis. Immunohistochemistry was performed using standard protocols and commercially available antibodies in an automated immunostainer (Ventana BenchMark, Tucson, AZ); 3, 3'diaminobenzidine was used as the chromogen and Harris's hematoxylin as the counterstain. The primary antibodies, sources and conditions used are reported in Table 1. All immunostainings were scored as a percentage of positive tumor cells by 3 pathologists (EK, MR, and SP). Moreover, p53 was considered with a mutated pattern when it was either completely negative (truncated pattern) or with $\geq 60 \%$-positive cell nuclei (missense pattern), or with a wildtype pattern in the remaining instances $[19,20]$. 
Table 1 Primary antibodies, sources, and conditions used in this study

\begin{tabular}{|c|c|c|c|c|c|c|c|}
\hline Antibody & Gene symbol & Clone & Species & Vendor & Address & Cat\# & Dilution \\
\hline c-myc & $M Y C$ & Y69 & Rabbit monoclonal & Ventana & Tucson, AZ & $790-4628$ & Predilute \\
\hline CD31 & PECAM1 & JC70 & Mouse & Ventana & Tucson, AZ & $760-4378$ & Predilute \\
\hline D2-40 & $P D P N$ & D2-40 & Mouse monoclonal & Ventana & Tucson, AZ & $760-4395$ & Predilute \\
\hline ERG & $E R G$ & EPR3864 & Rabbit monoclonal & Ventana & Tucson, AZ & $790-4398$ & Predilute \\
\hline ID1 & IDI & $195-14$ & Rabbit monoclonal & BioCheck & Foster city, CA & RN 38143 & $1: 50$ \\
\hline p16 & $C D K N 2 A$ & $\mathrm{E} 6 \mathrm{H} 4{ }^{\mathrm{TM}}$ & Mouse monoclonal & Ventana & Tucson, AZ & $705-4713$ & Predilute \\
\hline p53 & TP53 & DO-7 & Mouse monoclonal & Ventana & Tucson, AZ & 800-2912 & Predilute \\
\hline p63 & TP63 & $4 \mathrm{~A} 4$ & Mouse monoclonal & Ventana & Tucson, AZ & $790-4509$ & Predilute \\
\hline Pankeratin & NA & AE1/AE3/PCK26 & Mouse monoclonal & Ventana & Tucson, AZ & $760-2135$ & Predilute \\
\hline PAX8 & PAX8 & MRQ-50 & Mouse & Ventana & Tucson, AZ & $760-4618$ & Predilute \\
\hline Smooth muscle actin & ACTA2 & $1 \mathrm{~A} 4$ & Mouse monoclonal & Ventana & Tucson, AZ & $760-2833$ & Predilute \\
\hline Thyroglobulin & $T G$ & $2 \mathrm{H} 11+6 \mathrm{E} 1$ & Mouse monoclonal & Ventana-Roche & Tucson, AZ & $760-2671$ & prediluted \\
\hline TTF-1 & $T T F 1$ & 8G7G3/1 & Mouse monoclonal & Ventana & Tucson, AZ & $790-4398$ & Predilute \\
\hline
\end{tabular}

\section{Next-generation sequencing}

DNA extraction from formalin-fixed, paraffin embedded tissues was performed using Maxwell nucleic acid extractor (Promega, Italia S.R.L, Milan, Italy). NGS libraries were prepared, and the mutational status of 56 genes was analyzed using Myriapod ${ }^{\circledR}$ NGS-IL 56G Onco panel (Diatech Pharmacogenetics S.R.L., Jesi, Italy). Sequencing runs were performed by the MiSeq platform (Illumina) on a v2 cartridge $(2 \times 151)$ as previously described [21]. TERT promoter mutations were recently described as frequently occurring in the highly aggressive forms of thyroid cancer including anaplastic carcinomas [22, 23]. Thus, we explored the occurrence of TERT promoter mutations (C224T and C250T) in our set using a custom sequencing assay. Primary and secondary analyses of NGS data were performed by Myriapod NGS data analysis software (NG 900-SV, Diatech Pharmacogenetics S.R.L., Jesi, Italy).

Mutations were considered reliable with a minimum allele frequency of $5 \%$ and a minimum coverage of $\times 500$. Focusing on functional modifications, we excluded from the final list all non-coding mutations and considered only variants responsible for a missense, frameshift, deletion, or start/stop gain.

\section{Statistical analysis}

Analyses of comparison between immunohistochemical results and tumor types were performed by applying Fisher's exact test and Mann-Whitney test.

Analyses of association between the mutational status of genes and tumor types were performed using Fisher's exact test and generalized linear models. Data were considered statistically significant with a $p$-value $<0.05$. All statistical analyses were performed using R Software v 3.5.1.

\section{Results}

\section{Clinicopathologic features}

The clinicopathologic features of the 10 patients with angiosarcoma and of the 22 patients with anaplastic carcinoma are summarized in Table 2. Angiosarcoma presented in 6 males (aged 57-75 ys, mean age 69 ys) and 4 females (aged 52-91 ys, mean age 70 ys), while anaplastic carcinoma presented in 9 men (aged 39-80 ys, mean age 65 ys) and in 13 women (aged 52-84 ys, mean age 69 ys).

Follow-up was available for 6 angiosarcoma patients (2-66 months, mean follow-up 16 months) and for 16 anaplastic carcinoma patients (1-194 months, mean followup 34 months). Five (83\%) out of 6 angiosarcoma patients died from the thyroid neoplasia, while one $(17 \%)$ is still alive with metastatic disease in the lung after 66 months follow-up. Twelve $(75 \%)$ out of 16 anaplastic carcinoma patients died because of the disease, 3 (19\%) died from other causes (myocardial infarction in two cases and cerebral glioma in one case) and one patient (6\%) is still alive with no evidence of disease after 88 months follow-up. Interestingly, no angiosarcoma spread to regional or distant lymph nodes was present, while in 5 patients with anaplastic carcinoma nodal metastases were identified. No patient was known to have received prior radiation therapy.

Grossly, 7 (70\%) of 10 angiosarcomas presented as a hemorrhagic-necrotic mass with variable cystic changes. In $3(30 \%)$ of 10 cases, the lesion was described as a bloody cyst with a variable wall thickness (Fig. 1). The tumor size ranged from 4.5 to $12 \mathrm{~cm}$. Histologically, all the cases were composed of a neoplastic proliferation of atypical cells, with enlarged pleomorphic nuclei and prominent nucleoli, arranged in sheets and lining vascular spaces. In 6 cases $(60 \%)$ the epithelioid appearance was prevalent, while in 
Table 2 Clinicopathological features of 10 angiosarcomas and 22 anaplastic carcinomas of the thyroid

\begin{tabular}{|c|c|c|c|c|c|c|c|}
\hline & Sex & $\begin{array}{l}\text { Age at } \\
\text { surgery }\end{array}$ & $\begin{array}{l}\text { Prevalent histological } \\
\text { pattern }\end{array}$ & $\begin{array}{l}\text { Well-differentiated } \\
\text { component }\end{array}$ & Site of metastases & Treatment & $\begin{array}{l}\text { Follow-up, } \\
\text { months }\end{array}$ \\
\hline \multicolumn{8}{|c|}{ Angiosarcomas } \\
\hline 1 & M & 57 & Epithelioid & & & Thyroidectomy & NA \\
\hline 2 & M & 66 & Epithelioid & & Bone (femur) & Thyroidectomy, & DOD, 5 \\
\hline 3 & M & 71 & Sarcomatoid & & Neck & Thyroidectomy, & DOD, 5 \\
\hline 4 & M & 75 & Sarcomatoid & & Bone (femur) & hyroidectomy & NA \\
\hline 5 & M & 73 & Epithelioid & & Neck, lung & Thyroidectomy & DOD, 11 \\
\hline 6 & $\mathrm{~F}$ & 52 & Epithelioid & & Mediastinum & Thyroidectomy, chemotherapy & DOD, 9 \\
\hline 7 & $\mathrm{~F}$ & 91 & Epithelioid & & Neck & Thyroidectomy & DOD, 2 \\
\hline 8 & $\mathrm{~F}$ & 71 & Epithelioid & & & Thyroidectomy & NA \\
\hline 9 & M & 74 & Sarcomatoid & & Lung & Thyroidectomy, chemotherapy & AWD, 66 \\
\hline 10 & $\mathrm{~F}$ & 68 & Sarcomatoid & & & Throidectomy & NA \\
\hline \multicolumn{8}{|c|}{ Anaplastic carcinomas } \\
\hline 1 & M & 72 & Squamoid & & & Thyroidectomy & NA \\
\hline 2 & M & 58 & Sarcomatoid & $\begin{array}{l}\text { Papillary carcinoma, tall } \\
\text { cell variant }\end{array}$ & & Thyroidectomy & NA \\
\hline 3 & M & 67 & Pleomorphic epithelioid & $\begin{array}{l}\text { Classic papillary } \\
\text { carcinoma }\end{array}$ & & Thyroidectomy & DOC, 78 \\
\hline 4 & $\mathrm{~F}$ & 74 & Sarcomatoid & & & Thyroidectomy & NA \\
\hline 5 & $\mathrm{~F}$ & 84 & $\begin{array}{l}\text { Pleomorphic epithelioid } \\
\text { with giant cells }\end{array}$ & $\begin{array}{l}\text { Papillary carcinoma, tall } \\
\text { cell variant }\end{array}$ & & Thyroidectomy & NA \\
\hline 6 & M & 39 & Pleomorphic epithelioid & Hürthle cell carcinoma & & Thyroidectomy & NA \\
\hline 7 & M & 59 & Pleomorphic epithelioid & $\begin{array}{l}\text { Papillary carcinoma, tall } \\
\text { cell variant }\end{array}$ & & Thyroidectomy & DOD, 1 \\
\hline 8 & $\mathrm{~F}$ & 70 & $\begin{array}{l}\text { Pleomorphic epithelioid } \\
\text { with giant cells }\end{array}$ & Hürthle cell carcinoma & & Thyroidectomy & DOD, 1 \\
\hline 9 & $\mathrm{~F}$ & 73 & Pleomorphic epithelioid & $\begin{array}{l}\text { Papillary carcinoma, } \\
\text { follicular variant }\end{array}$ & & Thyroidectomy & DOC, 194 \\
\hline 10 & M & 69 & Sarcomatoid & $\begin{array}{l}\text { Papillary carcinoma, } \\
\text { follicular variant }\end{array}$ & & Thyroidectomy & DOD, 2 \\
\hline 11 & $\mathrm{~F}$ & 52 & Sarcomatoid & Hürthle cell carcinoma & & Thyroidectomy & DOC, 100 \\
\hline 12 & $\mathrm{~F}$ & 63 & $\begin{array}{l}\text { Pleomorphic epithelioid } \\
\text { with giant cells }\end{array}$ & & Lung & Thyroidectomy & DOD, 1 \\
\hline 13 & $\mathrm{~F}$ & 65 & Sarcomatoid & & Neck, mediastinum & Thyroidectomy & DOD, 1 \\
\hline 14 & M & 47 & Pleomorphic epithelioid & Hürthle cell carcinoma & $\begin{array}{l}\text { Lung, pleura, lymph } \\
\text { nodes }\end{array}$ & $\begin{array}{l}\text { Thyroidectomy, radiotherapy, } \\
\text { chemotherapy }\end{array}$ & DOD, 22 \\
\hline 15 & M & 80 & $\begin{array}{l}\text { Pleomorphic epithelioid } \\
\text { with giant cells }\end{array}$ & & Lung, neck & Thyroidectomy, radiotherapy & DOD, 2 \\
\hline 16 & $\mathrm{~F}$ & 82 & $\begin{array}{l}\text { Sarcomatoid with giant } \\
\text { cells }\end{array}$ & & Lung, & Thyroidectomy & DOD, 2 \\
\hline 17 & M & 78 & Squamoid & $\begin{array}{l}\text { Papillary carcinoma, tall } \\
\text { cell variant }\end{array}$ & $\begin{array}{l}\text { Lung, pleura, bone, } \\
\text { lymph nodes }\end{array}$ & Thyroidectomy & DOD, 4 \\
\hline 18 & M & 74 & Squamoid & & $\begin{array}{l}\text { Neck, oropharynx, } \\
\text { lymph nodes }\end{array}$ & Thyroidectomy, radiotherapy & DOD, 9 \\
\hline 19 & $\mathrm{~F}$ & 63 & Pleomorphic epithelioid & & & $\begin{array}{l}\text { Thyroidectomy, radiotherapy, } \\
\text { radiometabolic therapy }\end{array}$ & ANED, 88 \\
\hline 20 & $\mathrm{~F}$ & 84 & Sarcomatoid & $\begin{array}{l}\text { Papillary carcinoma, } \\
\text { columnar cell variant }\end{array}$ & $\begin{array}{l}\text { Lung, lymph nodes, } \\
\text { bone }\end{array}$ & Thyroidectomy, radiotherapy & DOD, 3 \\
\hline 21 & $\mathrm{~F}$ & 74 & Sarcomatoid & & & Thyroidectomy & NA \\
\hline 22 & $\mathrm{~F}$ & 56 & Pleomorphic epithelioid & & Lung, lymph nodes & Thyroidectomy & DOD, 3 \\
\hline
\end{tabular}

$N A$ not available, $D O D$ dead of disease, $D O C$ dead of other causes, $A W D$ alive with disease, $A N E D$ alive with no evidence of disease

the remaining 4 cases (40\%), the proliferation showed mainly a sarcomatous growth pattern, with neoplastic spindle cells arranged in irregular fascicles. In all cases, however, these two patterns were intermingled with each other, at least focally, and necrotic areas were present. In all 3 cystic cases, neoplastic cells were identifiable only at the periphery of the lesion. In no angiosarcoma was a welldifferentiated thyroid neoplasia identified.
Macroscopically, all anaplastic carcinomas were described as largely lobulated and partially necrotic solid mass, ranging from 5 to $10 \mathrm{~cm}$ in size, often extending to perithyroidal soft tissues (Fig. 1). Histologically, they were characterized by three main growth patterns: pleomorphic epithelioid, squamoid and spindle cell/sarcomatoid, which were sometimes combined at the same time with or without osteoclast-like giant cells. In $12(54 \%)$ of 22 cases, a well- 

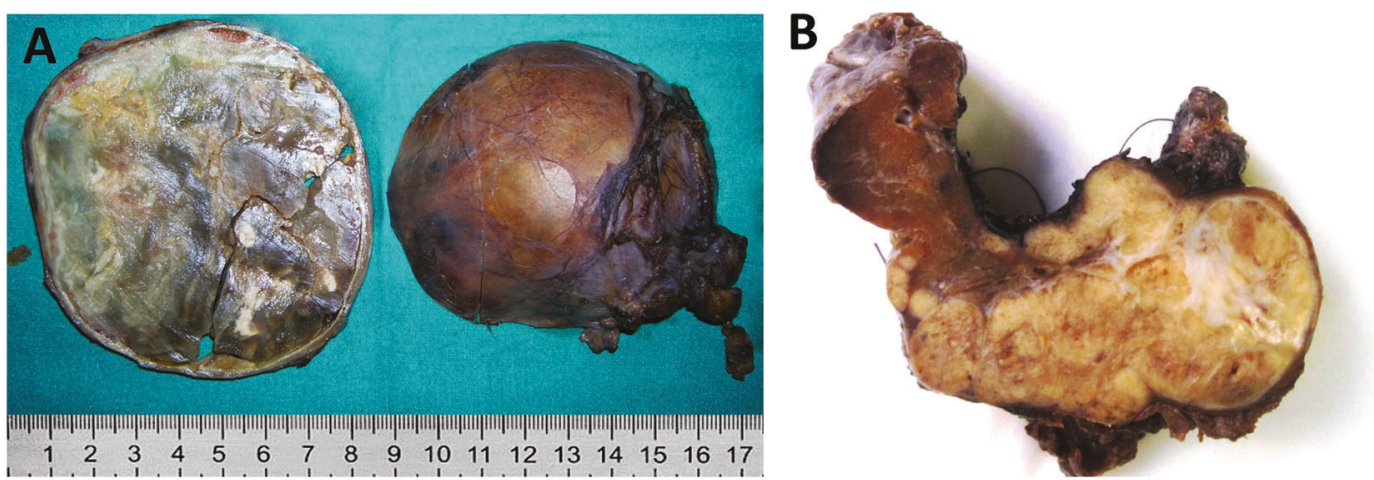

Fig. 1 Macroscopic features. Grossly, angiosarcoma presents as a large cyst, filled with necrotic and hemorrhagic material a, while anaplastic carcinoma mainly presents as a solid mass with variable necrosis $\mathbf{b}$

differentiated tumor component (papillary carcinoma in 8 cases and Hürthle cell carcinoma in 4 cases) was recognizable, whereas in the remaining $10(45 \%)$ cases, the whole neoplasia was histologically anaplastic. In $2(9 \%)$ of 22 cases, the tumor was extensively necrotic, and the anaplastic proliferation was limited to some peripheral areas.

Two cases (cases 21 and 22), originally diagnosed as angiosarcomas, have been reinterpreted as anaplastic carcinomas during pathological review. Histologically, case 21 was a spindle cell solid proliferation of neoplastic cells with wide necrotic areas and case 22 was composed of a proliferation of epithelioid cells with hobnail features, arranged in vascular-like spaces devoid of red cells (see below).

\section{Immunohistochemical features}

The expression of several immunomarkers was investigated in both angiosarcomas and anaplastic carcinomas by searching for potential differences with a diagnostic meaning. The immunohistochemical results are summarized in Table 3.

All analyzed angiosarcomas showed a consistent and diffuse positivity for vascular markers CD31, ERG, and ID1, while D2-40 was positive in only 1 (10\%) angiosarcoma. On the other hand, among vascular markers, both D2-40 and ID1 were variably positive in 55\% of analyzed anaplastic carcinomas, irrespectively of their growth pattern. Moreover, about $5 \%$ of neoplastic cells stained with ERG in $1(5 \%)$ anaplastic carcinoma with an epithelioid pattern and only rare cells stained with CD31 in 2 (9\%) anaplastic carcinomas with a sarcomatoid prevalence.

Among the markers of thyroid lineage, thyroglobulin was negative in all anaplastic carcinomas, while PAX8 and TTF-1 showed some positivity in anaplastic carcinomas. Specifically, PAX8 was focally detected in 11 anaplastic carcinoma samples $(50 \%)$, all with the epithelioid or squamoid pattern of growth, whereas TTF-1 was focally positive in only 1 epithelioid anaplastic carcinoma sample (5\%). No anaplastic carcinomas with a prevalent sarcomatoid pattern stained with these markers of thyroid origin. Notably, all these markers were absent in angiosarcomas.

Pankeratin was positive in most angiosarcomas and anaplastic carcinomas (70 and $82 \%$ of cases, respectively) with a high variability of cell percentage. It tended to stain more than $50 \%$ of the tumoral cells in epithelioid and squamoid anaplastic carcinomas, while in sarcomatoid anaplastic carcinomas the percentage of positive cells was much lower (average 10\%). In addition, p63 was expressed in $12(55 \%)$ of 22 anaplastic carcinomas with a more diffuse staining in squamoid or epithelioid anaplastic carcinomas and only in isolated cells in $1(10 \%)$ of 10 angiosarcomas.

Similarly, smooth muscle actin (SMA) was negative in all angiosarcomas but showed a variable reactivity in anaplastic carcinomas. The cell cycle regulator p16 was positive in $78 \%$ of angiosarcomas and in $41 \%$ of anaplastic carcinomas. Interestingly, c-myc positivity was found in approximately half of the cases of both angiosarcomas and anaplastic carcinomas. Finally, p53 showed a wild-type pattern in all angiosarcomas and a mutated pattern in 15 (71\%) of 22 anaplastic carcinomas. The most useful immunomarkers supporting either a angiosarcoma or a anaplastic carcinoma diagnosis are shown in Fig. 2.

The reviewed cases (cases 21 and 22), originally diagnosed as angiosarcomas, showed a focal immunoreactivity for pankeratin, c-myc and D2-40, but negativity for all the other endothelial markers (CD31, ERG, and ID1) (Fig. 3).

\section{Genetic features}

Only 4 (40\%) of 10 angiosarcomas and 17 (77\%) of 22 anaplastic carcinomas yielded DNA of quality and quantity sufficient to perform genetic analysis. The low yield was likely dependent on the age of the samples.

To compare the genetic profile of angiosarcomas and anaplastic carcinomas, we used the NGS approach to sequence hotspot regions within a panel of 56 genes that have been found recurrently mutated in several cancers. Moreover, TERT promoter mutations were sequenced using 
Table 3 Comparison of immunohistochemical results between 10 angiosarcomas and 22 anaplastic carcinomas of the thyroid

\begin{tabular}{|c|c|c|c|c|}
\hline Antibodies & $n(\%)$ & Angiosarcomas & Anaplastic carcinomas & $p$-value \\
\hline c-myc, total & 32 & 10 & 22 & n.s.^ \\
\hline Negative $(<1 \%)$ & $17(53)$ & $5(50)$ & $12(55)$ & \\
\hline Positive $(\geq 1 \%)$ & $15(47)$ & $5(50)$ & $10(45)$ & \\
\hline c-myc mean (range) & $15 \%(0-90 \%)$ & $17 \%(0-90 \%)$ & $14 \%(0-80 \%)$ & n.s.* \\
\hline CD31, total & 32 & 10 & 22 & $<0.0001^{\wedge}$ \\
\hline Negative $(<1 \%)$ & $20(63)$ & $0(0)$ & $20(91)$ & \\
\hline Positive ( $\geq 1 \%)$ & $12(37)$ & $10(100)$ & $2(9)$ & \\
\hline CD31 mean (range) & $30 \%(0-100 \%)$ & $93 \%(60-100 \%)$ & $1 \%(0-20 \%)$ & $<0.0001 *$ \\
\hline D2-40, total & 32 & 10 & 22 & $0.0237^{\wedge}$ \\
\hline Negative $(<1 \%)$ & $19(59)$ & $9(90)$ & $10(45)$ & \\
\hline Positive $(\geq 1 \%)$ & $13(41)$ & $1(10)$ & $12(55)$ & \\
\hline D2-40 mean (range) & $16 \%(0-100 \%)$ & $9 \%(0-90 \%)$ & $19 \%(0-100 \%)$ & 0.0419* \\
\hline ERG, total & 31 & 9 & 22 & $<0.0001^{\wedge}$ \\
\hline Negative $(<1 \%)$ & $21(68)$ & $0(0)$ & $21(95)$ & \\
\hline Positive $(\geq 1 \%)$ & $10(32)$ & $9(100)$ & $1(5)$ & \\
\hline ERG mean (range) & $26 \%(0-100 \%)$ & $92 \%(70-100 \%)$ & $0 \%(0-5 \%)$ & $<0.0001 *$ \\
\hline ID1, total & 26 & 6 & 20 & n.s. ${ }^{\wedge}$ \\
\hline Negative $(<1 \%)$ & $9(35)$ & $0(0)$ & $9(45)$ & \\
\hline Positive $(\geq 1 \%)$ & $17(65)$ & $6(100)$ & $11(55)$ & \\
\hline ID1 mean (range) & $37 \%(0-100 \%)$ & $86 \%(60-100 \%)$ & $23 \%(0-100 \%)$ & $0.005^{*}$ \\
\hline p16, total & 31 & 9 & 22 & n.s.^ \\
\hline Negative $(<1 \%)$ & $15(48)$ & $2(22)$ & $13(59)$ & \\
\hline Positive $(\geq 1 \%)$ & $16(52)$ & $7(78)$ & $9(41)$ & \\
\hline p16 mean (range) & $21 \%(0-100 \%)$ & $30 \%(0-50 \%)$ & $17 \%(0-100 \%)$ & n.s. \\
\hline $\mathrm{p} 53^{+}$, total & 31 & 10 & 21 & $0.0002^{\wedge}$ \\
\hline Wild-type pattern & $16(52)$ & $10(100)$ & $6(29)$ & \\
\hline Mutated pattern & $15(48)$ & $0(0)$ & $15(71)$ & \\
\hline p53 mean (range) & $34 \%(0-100 \%)$ & $21 \%(1-40 \%)$ & $40 \%(0-100 \%)$ & n.s. \\
\hline p63, total & 32 & 10 & 22 & $0.0237^{\wedge}$ \\
\hline Negative $(<1 \%)$ & $19(59)$ & $9(90)$ & $10(45)$ & \\
\hline Positive $(\geq 1 \%)$ & $13(41)$ & $1(10)$ & $12(55)$ & \\
\hline p63 mean (range) & $18 \%(0-100 \%)$ & $0 \%(0-1 \%)$ & $27 \%(0-100 \%)$ & 0.0153* \\
\hline Pankeratin, total & 32 & 10 & 22 & n.s. ${ }^{\wedge}$ \\
\hline Negative $(<1 \%)$ & $7(22)$ & $3(30)$ & $4(18)$ & \\
\hline Positive ( $\geq 1 \%)$ & $25(78)$ & $7(70)$ & $18(82)$ & \\
\hline Pankeratin mean (range) & $36 \%(0-100 \%)$ & $36 \%(0-90 \%)$ & $35 \%(0-100 \%)$ & n.s. \\
\hline PAX8, total & 32 & 10 & 22 & $0.006^{\wedge}$ \\
\hline Negative $(<1 \%)$ & $21(66)$ & $10(100)$ & $11(50)$ & \\
\hline Positive $(\geq 1 \%)$ & $11(34)$ & $0(0)$ & $11(50)$ & \\
\hline PAX8 mean (range) & $14 \%(0-100 \%)$ & $0 \%$ & $21 \%(0-100 \%)$ & $0.022^{*}$ \\
\hline SMA, total & 31 & 9 & 22 & $0.0297^{\wedge}$ \\
\hline Negative $(<1 \%)$ & $21(68)$ & $9(100)$ & $12(55)$ & \\
\hline Positive $(\geq 1 \%)$ & $10(32)$ & $0(0)$ & $10(45)$ & \\
\hline SMA mean (range) & $14 \%(0-100 \%)$ & $0 \%$ & $19 \%(0-100 \%)$ & n.s.* \\
\hline TTF-1, total & 32 & 10 & 22 & n.s.^ \\
\hline Negative $(<1 \%)$ & $31(97)$ & $10(100)$ & $21(95)$ & \\
\hline Positive $(\geq 1 \%)$ & $1(3)$ & $0(0)$ & $1(5)$ & \\
\hline TTF-1 mean (range) & $0 \%(0-1 \%)$ & $0 \%$ & $0 \%(0-1 \%)$ & n.s.* \\
\hline
\end{tabular}

Total number of cases analyzed;

${ }^{\wedge}$ Fisher's test; *Mann-Whitney test; ${ }^{+}$p53 wild-type pattern, $1-59 \%$ of positive cells and p53 mutated pattern, either $0 \%$ or $60-100 \%$ of positive cells. Bold: statistically significant values $(p<0.05)$

a custom assay. We detected 503 variants within the set of analyzed samples. Of these, 111 were somatic, functional mutations. The vast majority of the identified mutations were missense variants (72\%) (Fig. 4a). Mutations were detected in 15 (26\%) of the 57 genes analyzed in this study (Table 4 and Fig. 4b). All but one analyzed sample presented mutations in at least one gene, and $16(76 \%)$ of 21 samples showed a co-occurrence of at least 2 or more 
Fig. 2 Microscopic features of angiosarcomas (left panel) and anaplastic carcinomas (right panel). They both show an epithelioid morphology and are positive for cytokeratin (CK). Angiosarcoma presents a strong and diffuse cytoplasmic positivity for CD31 and a nuclear positivity for ERG, whereas anaplastic carcinoma is negative for both endothelial markers and it is characterized by 53 positivity in $70 \%$ of neoplastic cells (mutated pattern) and focal positivity for PAX8
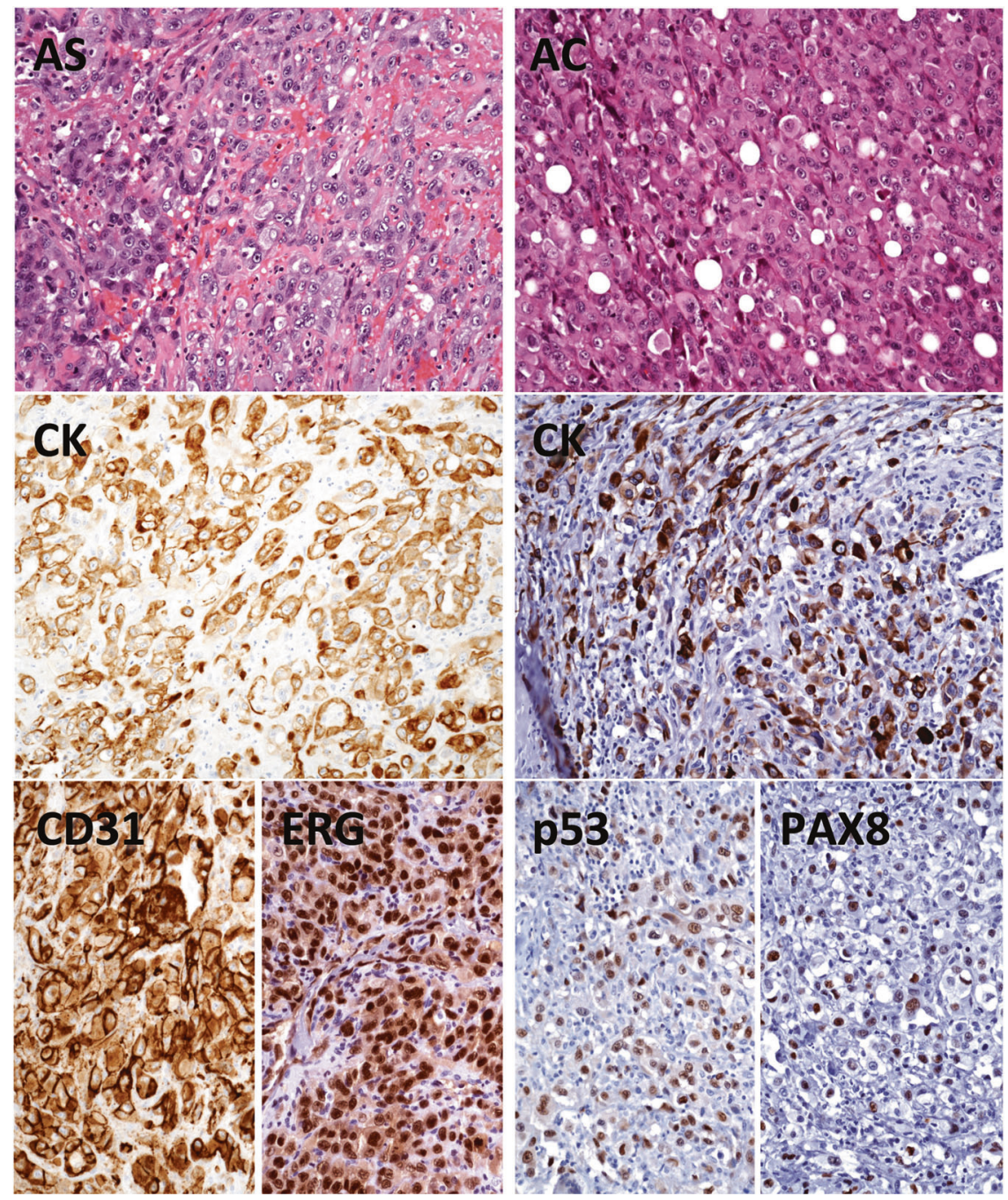

mutated genes (Fig. 4c). The average total number of mutations per sample was 3.0 and there was a significantly lower mutational load in angiosarcomas compared to anaplastic carcinomas ( $1 \pm 0.8$ vs $3.2 \pm 1.7, p$-value 0.022$)$. The top three mutated genes were TP53, TERT, and PIK3CA, which were found mutated in 13, 10, and 6 different cases, respectively (Fig. 4b). Among the 13 cases with TP53 somatic mutations, $8(62 \%)$ harbored missense mutations, and the remaining $5(38 \%)$ cases harbored truncating mutations (3 frameshift and 2 nonsense). Notably, TP53 mutations were found exclusively in anaplastic carcinomas, whereas no TP53 mutation was detected in angiosarcomas ( $p$-value 0.0172) (Fig. 4d). There was a significant correlation between the presence of TP53 mutation and the mutant pattern of p53 immunostaining ( $p$-value 0.0001).

Furthermore, TERT promoter mutations were found in 10 (59\%) of 17 anaplastic carcinomas, but not in angiosarcomas ( $p$-value: 0.0902). All TERT mutations were $-124 \mathrm{c}>\mathrm{t}$ (C228T). No association was found between TP53 and TERT mutations in anaplastic carcinomas.
No statistically significant differences were found between angiosarcomas and anaplastic carcinomas for the other genes (Table 4 and Fig. 4).

\section{Discussion}

Thyroid angiosarcomas and anaplastic carcinomas have been traditionally considered strictly related due to their undifferentiated, sarcomatous morphologic appearance and the shared immunohistochemical expression of cytokeratin. Their rarity among thyroid tumors and a similar well-known dismal prognosis encouraged considering the distinction merely academic.

Indeed, both angiosarcomas and anaplastic carcinomas usually present with a rapidly growing thyroid mass, causing symptoms related to mechanical compression and invasion of adjacent structures. Patients with angiosarcoma and anaplastic carcinoma carry an ominous prognosis, although it has been reported that patients with localized 
Fig. 3 One case (case 22) originally diagnosed as angiosarcoma has been reclassified anaplastic carcinoma after pathological review. Histologically, it represents a sort of "missing-link" between these two entities as it is composed by a proliferation of epithelioid cells with hobnail features, arranged in vascularlike spaces. These spaces are devoid of red cells, an important clue for differential diagnosis (HE, a). Immunohistochemical positivity for cytokeratin b, PAX8 c, p63 d, and p53 e, along with negativity for CD31 f, allow for the correct diagnosis
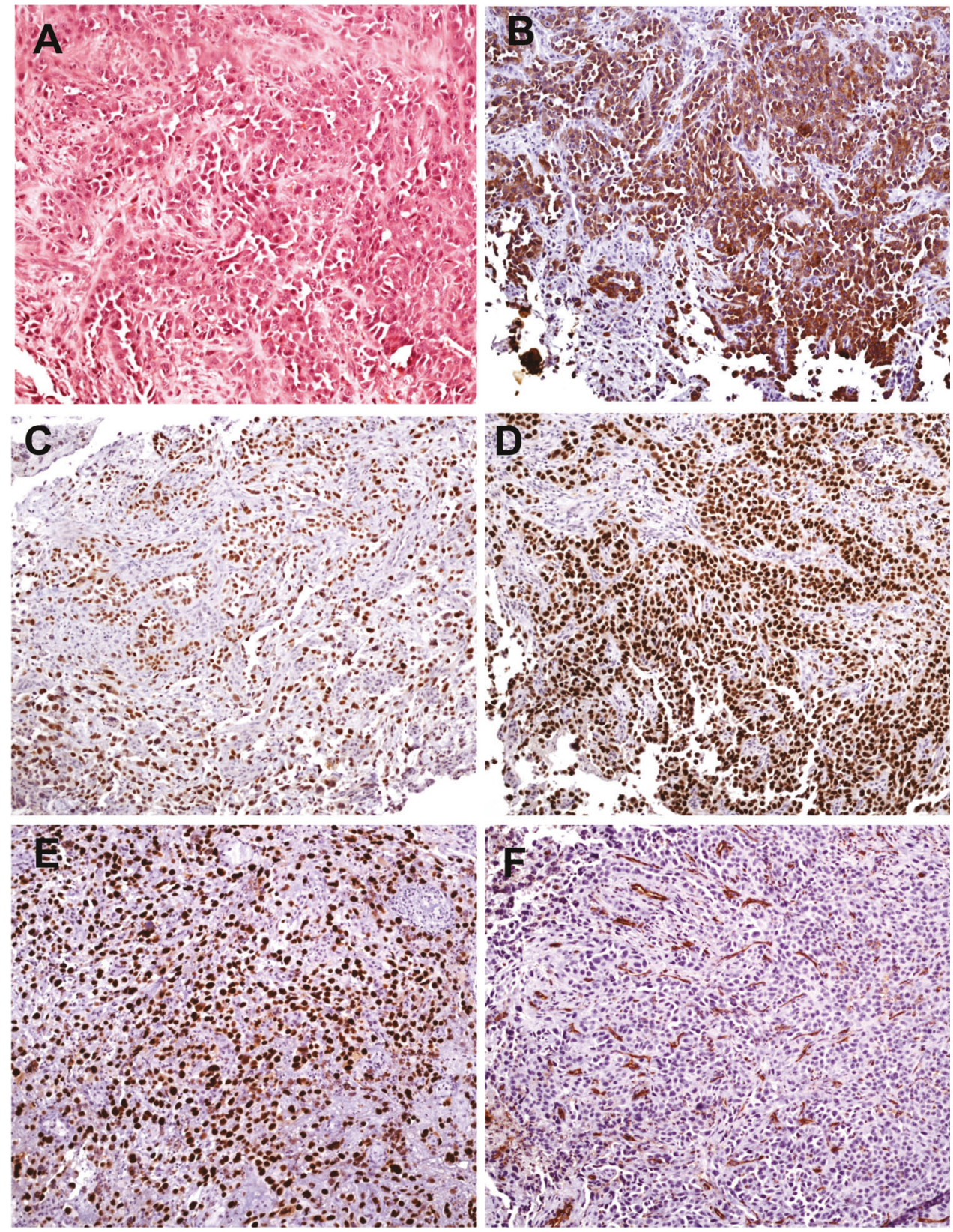

disease at diagnosis can be associated with prolonged survival [24-26]. In our study, two patients are experiencing a prolonged survival, one with anaplastic carcinoma (case 19, Table 2), who is alive with no evidence of disease after an 88 months follow-up, and one angiosarcoma patient (case 9, Table 2), who is alive with lung metastasis after a 66 months follow-up.

Surgical resection is the recommended primary treatment in both angiosarcomas and anaplastic carcinomas. In cases of advanced disease, adjuvant chemotherapy and/or radiotherapy may be used [27]. The rarity of these lesions together with their limited genetic and molecular characterization have so far hampered the development of tailored treatment protocols, leaving patients with inadequate therapeutic options.

In this study, we aimed to compare morphologic, immunohistochemical and genetic features of a series of surgically resected angiosarcomas and anaplastic carcinomas in order to (1) deepen the knowledge of the molecular and genetic features associated with these thyroid cancer subtypes, and (2) obtain clues by a comparison of the characteristics of these lesions that would answer the question regarding whether these lesions share a common origin or are completely different entities.

In our experience, the macroscopic presentation of angiosarcoma was mainly as a cystic, necrotic and 


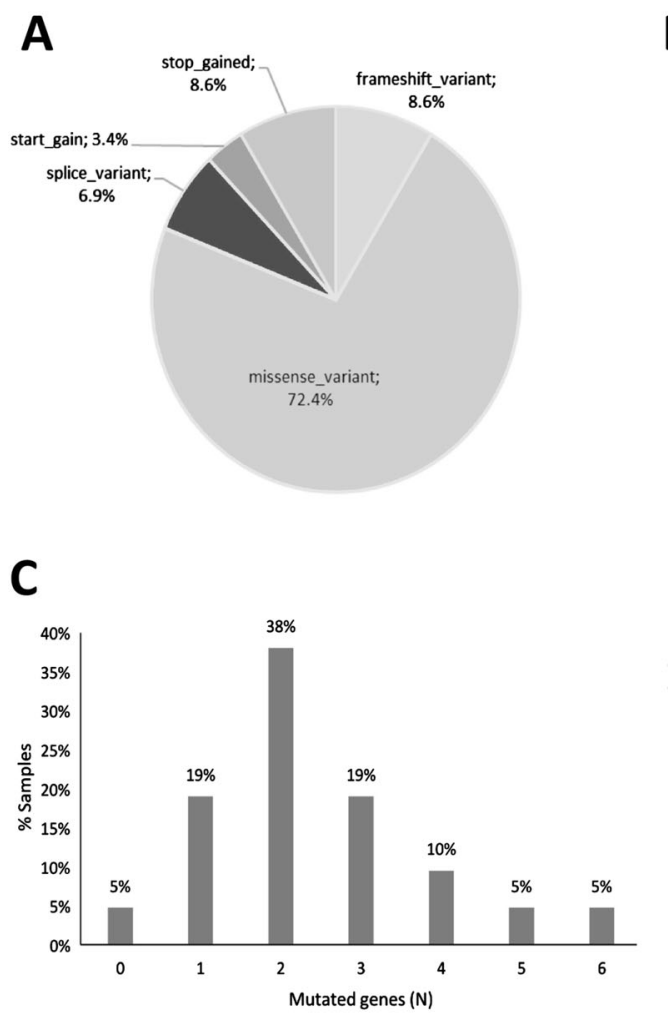

Fig. 4 Mutational profile of angiosarcomas and anaplastic carcinomas. Type of mutation according to the type of nucleotide substitution a. Percentage of mutated samples for each gene b. Frequency of

hemorrhagic nodule, whereas anaplastic carcinoma was typically a solid mass with variable necrosis (Fig. 1). Histologically, epithelioid angiosarcomas are quite similar to epithelioid pleomorphic anaplastic carcinomas. Sheets of epithelioid atypical cells, with or without necrotic areas, tend to destroy thyroid parenchyma and extend to perithyroidal tissue in both neoplasias. On the other hand, sarcomatoid anaplastic carcinomas are constituted by spindle cells fascicles, reminiscent of sarcomatous proliferations. Importantly, both squamoid cells, which could be highlighted by p63 immunohistochemistry, and giant osteoclastlike cells were only associated with anaplastic carcinoma and never found in angiosarcomas.

To accurately diagnose these tumors, immunohistochemical stainings are thus mandatory and should include markers of endothelial differentiation, thyroid lineage differentiation and epithelial markers. Among endothelial markers, all our angiosarcomas were positive for CD31, ERG, and ID1, while D2-40 (podoplanin) was found in only one case of angiosarcoma; in contrast, anaplastic carcinomas showed immunoreactivity with D2-40 in at least half of the cases. This finding is not surprising as D2-40 is not exclusively an endothelial marker, but it is also known for its role in promoting epithelial-mesenchymal transition and
ANAPLASTIC CARCINOMAS $\square$ ANGIOSARCOMAS

mutation number in the entire cohort c. Histogram representing the percentage of mutated samples for the indicated genes according to angiosarcoma and anaplastic carcinoma diagnosis $\mathbf{d}$

tumor progression [28]. However, no correlation was seen among D2-40 positivity in anaplastic carcinomas and the presence of a well-differentiated component. Similarly, the high incidence of positivity observed for ID1, in both angiosarcomas and anaplastic carcinomas, highlights a possible role of this factor in the genesis and progression of these lesions. Coherently, it is known that the transcriptional regulator ID1 is associated with aggressive features in thyroid cancer, where it promotes the acquisition of mesenchymal-like features and the activation of the epithelial-mesenchymal process $[29,30]$.

Based on our results, PAX8 is the most reliable marker of thyroid origin in anaplastic carcinomas, being positive in $50 \%$ of cases, while TTF- 1 reactivity is found in only $5 \%$ of cases, and thyroglobulin is always negative. Similarly, p63 positivity should lean toward anaplastic carcinomas, since we found it in $~ 50 \%$ of anaplastic carcinomas and in only $10 \%$ of angiosarcomas. In contrast, pankeratin reacts with $82 \%$ of anaplastic carcinomas and $70 \%$ of angiosarcomas; therefore, it is not useful in the differential diagnosis between these two entities.

P16 was positive in $41 \%$ of anaplastic carcinomas, a result that confirms the involvement of cell cycle deregulation in thyroid follicular tumorigenesis and the 
Table 4 Comparison of detected mutations in thyroid angiosarcomas and anaplastic carcinomas

\begin{tabular}{|c|c|c|c|}
\hline & $\begin{array}{l}\text { Anaplastic carcinoma } \\
N(\%)\end{array}$ & $\begin{array}{l}\text { Angiosarcoma } \\
N(\%)\end{array}$ & $p$-value \\
\hline & 17 & 4 & \\
\hline$A P C$ & & & 1 \\
\hline Wild type & $16(94.1)$ & $4(100)$ & \\
\hline Mutated & $1(5.9)$ & $0(0)$ & \\
\hline ATM & & & 1 \\
\hline Wild type & $14(82.4)$ & $4(100)$ & \\
\hline Mutated & 3 (17.6) & $0(0)$ & \\
\hline$B R A F$ & & & 1 \\
\hline Wild type & 14 (82.4) & $4(100)$ & \\
\hline Mutated & $3(17.6)$ & $0(0)$ & \\
\hline$C D K N 2 A$ & & & 1 \\
\hline Wild type & $16(94.1)$ & $4(100)$ & \\
\hline Mutated & $1(5.9)$ & $0(0)$ & \\
\hline$H R A S$ & & & 1 \\
\hline Wild type & $13(76.5)$ & $3(75)$ & \\
\hline Mutated & $4(23.5)$ & $1(25)$ & \\
\hline KIT & & & 0.532 \\
\hline Wild type & $12(70.6)$ & $4(100)$ & \\
\hline Mutated & $5(29.4)$ & $0(0)$ & \\
\hline KRAS & & & 1 \\
\hline Wild type & $15(88.2)$ & $4(100)$ & \\
\hline Mutated & $2(11.8)$ & $0(0)$ & \\
\hline$M A P 2 K 1$ & & & 0.352 \\
\hline Wild type & $16(94.1)$ & $3(75)$ & \\
\hline Mutated & $1(5.9)$ & $1(25)$ & \\
\hline NRAS & & & 1 \\
\hline Wild type & $16(94.1)$ & $4(100)$ & \\
\hline Mutated & $1(5.9)$ & $0(0)$ & \\
\hline PDGFRA & & & 1 \\
\hline Wild type & $16(94.1)$ & $4(100)$ & \\
\hline Mutated & $1(5.9)$ & $0(0)$ & \\
\hline PIK3CA & & & 1 \\
\hline Wild type & $12(70.6)$ & $3(75)$ & \\
\hline Mutated & $5(29.4)$ & $1(25)$ & \\
\hline PTEN & & & 1 \\
\hline Wild type & $14(82.4)$ & $4(100)$ & \\
\hline Mutated & 3 (17.6) & $0(0)$ & \\
\hline$R B 1$ & & & 1 \\
\hline Wild type & $16(94.1)$ & $4(100)$ & \\
\hline Mutated & $1(5.9)$ & $0(0)$ & \\
\hline TERT & & & 0.090 \\
\hline Wild type & 7 (41.2) & $4(100.0)$ & \\
\hline Mutated & $10(58.8)$ & $0(0)$ & \\
\hline TP53 & & & 0.012 \\
\hline Wild type & $4(23.5)$ & $4(100)$ & \\
\hline Mutated & $13(76.5)$ & $0(0)$ & \\
\hline
\end{tabular}

Bold: statistically significant values $(p<0.05)$

progression from well-differentiated to anaplastic carcinoma [31, 32]. A role in neoplastic progression is ascertained also for c-myc, which has almost the same rate of positivity in anaplastic carcinomas and angiosarcomas. In addition to its already accepted diagnostic role in angiosarcomas [33], c-myc immunoreactivity has been reported in thyroid carcinomas with a growing expression in the undifferentiated components [34], and it is considered a potential target for personalized therapy [35].

In summary, based on our immunohistochemical findings, CD31 and ERG were the most reliable endothelial markers for angiosarcomas, while D2-40 focal positivity, without a convincing positivity for the previous markers, argued strongly against angiosarcomas as it was consistently positive in anaplastic carcinomas. Moreover, immunohistochemical positivity, even focal, for smooth muscle actin, p63, and PAX8 favored a diagnosis of anaplastic carcinoma. Last but not least, a mutated pattern (diffuse positivity or complete negativity) of p53 immunostaining prompted the same diagnosis.

In recent years, comprehensive genomic profiling using next-generation sequencing has provided novel information on the genetic alterations harbored by anaplastic carcinomas. In particular, anaplastic carcinomas are characterized by a high degree of genomic aberrations, including both a high mutational burden and copy number alterations [23, 36-39]. Even if anaplasti carcinoma shares some common mutations with well-differentiated thyroid cancers (such as the BRAF V600E), these lesions seem to be characterized by a distinctive set of alterations. Specifically, TP53 mutations have been shown to occur exclusively and with a very high frequency in anaplastic carcinomas, in line with the elevated genomic instability that characterizes these lesions [23, 39]. Moreover, TERT promoter mutations have been preferentially found in anaplastic carcinomas but are also frequently associated with the widely metastatic forms of papillary thyroid cancer [22, 23, 40]. Our results are fully in line with previous reports as TP53 and TERT mutations were the most frequent genetic alterations found in our anaplastic carcinoma subset.

On the other hand, very little is known about the genetic landscape of thyroid angiosarcomas. In fact, even the most recent papers are focused on immunohistochemical characterizations [41] or RNA transcript in situ hybridization [16], disregarding the genetic alterations associated with this disease. In our study, only a few mutations were found in angiosarcomas, and all of them were single events. Notably, no mutation in either TP53 or the TERT promoter could be detected in angiosarcomas. Even though we could obtain genetic data only in a limited number of angiosarcomas, the lack of common mutations between angiosarcomas and anaplastic carcinomas is undeniable and supports a different genetic origin between these two neoplasias. Therefore, these data strongly suggest that, in spite of a shared sarcomatous morphology and a similar clinical aggressiveness, angiosarcomas and anaplastic carcinomas likely rely on a completely different set of genetic alterations during their evolution. Intriguingly, genetic studies of 
angiosarcomas arising in soft tissue or other organs showed that, similarly to anaplastic carcinomas, these tumors are characterized by frequent activation of the mitogen activated protein kinase pathway (MAPK), as well as the alterations in the telomere homeostasis linked to mutations in the ATRX gene [42, 43].

In conclusion, our study provides new insights to consider these two neoplasias as separated and distinct cancer entities based on morphological, immunohistochemical and genetic features. As a consequence, it is our opinion that angiosarcomas should not be further considered an extreme in the spectrum of anaplastic carcinomas, but rather represents an independent subtype of thyroid cancers that could benefit from a diversified treatment. Further analyses are needed to consolidate our observations and to define a more detailed picture of the genetic abnormalities that occur in angiosarcomas and concur to the definition of this entity.

\section{Compliance with ethical standards}

Conflict of interest The authors declare that they have no conflict of interest.

Publisher's note: Springer Nature remains neutral with regard to jurisdictional claims in published maps and institutional affiliations.

\section{References}

1. Wick MR, Eusebi V, Lamovec J, Ryska A. Angiosarcoma. In: Lloyd RV, Osamura RY, Klöppel G, Rosai J, editors. WHO Classification of tumors of endocrine organs, Lyon: IARC; 2017. p. $129-32$.

2. Eckert F, Schmid U, Gloor F, hedinger C. Evidence of vascular differentiation in anaplastic tumours of the thyroid--an immunohistological study. Virchows Arch. 1986;410:203-15.

3. Krisch K, Holzner JH, Kokoschka R, jakesz R, Niderle B, Roka R. Hemangioendothelioma of the thyroid gland-true endothelioma or anaplastic carcinoma? Pathol Res Pract. 1980;170:230-42.

4. Mills SE, Gaffey MJ, Watts JC, Swanson PE, Wick MR, LiVolsi VA et al. Angiomatoid carcinoma and 'angiosarcoma' of the thyroid gland. A spectrum of endothelial differentiation. Am J Clin Pathol. 1994;102:322-30.

5. Mills SE, Stallings RG, Austin MB. Angiomatoid carcinoma of the thyroid gland. Anaplastic carcinoma with follicular and medullary features mimicking angiosarcoma. Am J Clin Pathol. 1986;86:674-8.

6. Chan YF, Ma L, Boey JH, Yeung HY. Angiosarcoma of the thyroid. An immunohistochemical and ultrastructural study of a case in a Chinese patient. Cancer. 1986;57:2381-8.

7. Eusebi V, Carcangiu ML, Dina R, Rosai J. Keratin-positive epithelioid angiosarcoma of thyroid. A report of four cases. Am J Surg Pathol. 1990;14:737-47.

8. Lamovec J, Zidar A, Zidanik B. Epithelioid angiosarcoma of the thyroid gland. Report of two cases. Arch Pathol Lab Med. 1994;118:642-6.

9. Maiorana A, Collina G, Cesinaro AM, Fano RA, Eusebi V. Epithelioid angiosarcoma of the thyroid. Clinicopathological analysis of seven cases from non-Alpine areas. Virchows Arch. 1996;429:131-7.
10. Tanda F, Massarelli G, Bosincu L, Cossu A. Angiosarcoma of the thyroid: a light, electron microscopic and histoimmunological study. Hum Pathol. 1988;19:742-5.

11. Totsch M, Dobler G, Feichtinger H, Sandbichler P, Ladurner D, Schmid KW. Malignant hemangioendothelioma of the thyroid. Its immunohistochemical discrimination from undifferentiated thyroid carcinoma. Am J Surg Pathol. 1990;14:69-74.

12. Goh SG, Chuah KL, Goh HK, Chen YY. Two cases of epithelioid angiosarcoma involving the thyroid and a brief review of nonAlpine epithelioid angiosarcoma of the thyroid. Arch Pathol Lab Med. 2003;127:E70-73.

13. Rotellini M, Vezzosi V, Bianchi S. Epithelioid angiosarcoma of the thyroid: report of a case from an Italian non-alpine area and review of the literature. Endocr Pathol. 2015;26:152-6.

14. Ryska A, Ludvikova M, Szepe P, Böör A. Epithelioid haemangiosarcoma of the thyroid gland. Report of six cases from a non-Alpine region. Histopathology. 2004;44:40-46.

15. Papotti M, Arrondini M, Tavaglione V, Veltri A, Volante M. Diagnostic controversies in vascular proliferations of the thyroid gland. Endocr Pathol. 2008;19:175-83.

16. Papotti M, Volante M, Negro F, Eusebi V, Bussolati G. Thyroglobulin mRNA expression helps to distinguish anaplastic carcinoma from angiosarcoma of the thyroid. Virchows Arch. 2000;437:635-42.

17. Ritter JH, Mills SE, Nappi O, Wick MR. Angiosarcoma-like neoplasms of epithelial organs: true endothelial tumors or variants of carcinoma? Semin Diagn Pathol. 1995;12:270-82.

18. Smallridge RC, Marlow LA, Copland JA. Anaplastic thyroid cancer: molecular pathogenesis and emerging therapies. Endocr Relat Cancer. 2009;16:17-44.

19. Kuhn E, Kurman RJ, Vang R, Sehdev AS, Han G, Soslow R, et al. TP53 mutations in serous tubal intraepithelial carcinoma and concurrent pelvic high-grade serous carcinoma--evidence supporting the clonal relationship of the two lesions. J Pathol. 2012;226:421-6.

20. Kuhn E, Wu RC, Guan B, Wu G, Zhang J, Wang Y, et al. Identification of molecular pathway aberrations in uterine serous carcinoma by genome-wide analyses. J Natl Cancer Inst. 2012;104:1503-13.

21. Lococo F, Gandolfi G, Rossi G, Pinto C, Rapicetta C, Cavazza A, et al. Deep sequencing analysis reveals that KRAS mutation is a marker of poor prognosis in patients with pulmonary sarcomatoid carcinoma. J Thorac Oncol. 2016;11:1282-92.

22. Gandolfi G, Ragazzi M, de Biase D, Visani M, Zanetti E, Torricelli F, et al. Genome-wide profiling identifies the THYT1 signature as a distinctive feature of widely metastatic papillary thyroid carcinomas. Oncotarget. 2018;9:1813-25.

23. Landa I, Ibrahimpasic T, Boucai L, Sinha R, Knauf JA, Shah RH, et al. Genomic and transcriptomic hallmarks of poorly differentiated and anaplastic thyroid cancers. J Clin Invest. 2016;126:1052-66.

24. De Felice F, Moscatelli E, Orelli S, Bulzonetti N, Musio D, Tombolini V. Primary thyroid angiosarcoma: a systematic review. Oral Oncol. 2018;82:48-52.

25. Dibelius G, Mehra S, Clain JB, Urken ML, Wenig BM. Noninvasive anaplastic thyroid carcinoma: report of a case and literature review. Thyroid. 2014;24:1319-24.

26. Pichardo-Lowden A, Durvesh S, Douglas S, Todd W, Bruno M, Goldenberg D. Anaplastic thyroid carcinoma in a young woman: a rare case of survival. Thyroid. 2009;19:775-9.

27. Patel SH, Hayden RE, Hinni ML, Wong WW, Foote RL, Milani S, et al. Angiosarcoma of the scalp and face: the Mayo Clinic experience. JAMA Otolaryngol Head Neck Surg. 2015;141:335-40.

28. Krishnan H, Rayes J, Miyashita T, Ishii G, Retzbach EP, Sheehan SA, et al. Podoplanin: an emerging cancer biomarker and therapeutic target. Cancer Sci. 2018;109:1292-9. 
29. Ciarrocchi A, Piana S, Valcavi R, Gardini G, Casali B. Inhibitor of DNA binding-1 induces mesenchymal features and promotes invasiveness in thyroid tumour cells. Eur $\mathbf{J}$ Cancer. 2011;47:934-45.

30. Gugnoni M, Sancisi V, Gandolfi G, Manzotti G, Ragazzi M, Giordano D, et al. Cadherin-6 promotes EMT and cancer metastasis by restraining autophagy. Oncogene. 2017;36:667-77.

31. Ferru A, Fromont G, Gibelin H, Guilhot J, Savagner F, Tourani JM, et al. The status of CDKN2A alpha (p16INK4A) and beta (p14ARF) transcripts in thyroid tumour progression. Br J Cancer. 2006;95:1670-7.

32. Pita JM, Figueiredo IF, Moura MM, Leite V, Cavaco BM. Cell cycle deregulation and TP53 and RAS mutations are major events in poorly differentiated and undifferentiated thyroid carcinomas. J Clin Endocrinol Metab. 2014;99:E497-507.

33. Ginter PS, Mosquera JM, MacDonald TY, D'Alfonso TM, Rubin MA, Shin SJ. Diagnostic utility of MYC amplification and antiMYC immunohistochemistry in atypical vascular lesions, primary or radiation-induced mammary angiosarcomas, and primary angiosarcomas of other sites. Hum Pathol. 2014;45:709-16.

34. Sakr HI, Chute DJ, Nasr C, Sturgis CD. cMYC expression in thyroid follicular cell-derived carcinomas: a role in thyroid tumorigenesis. Diagn Pathol. 2017;12:71.

35. Enomoto K, Zhu X, Park S, Zhao L, Zhu YJ, Willingham MC, et al. Targeting MYC as a therapeutic intervention for anaplastic thyroid cancer. J Clin Endocrinol Metab. 2017;102:2268-80.

36. Bonhomme B, Godbert Y, Perot G, Al Ghuzlan A, Bardet S, Belleannée G, al. Molecular pathology of anaplastic thyroid carcinomas: a retrospective study of 144 cases. Thyroid. 2017;27:682-92.
37. Collini P, Barisella M, Renne SL, Pizzi N, Mattavelli D, Stacchiotti S, et al. Epithelioid angiosarcoma of the thyroid gland without distant metastases at diagnosis: report of six cases with a long follow-up. Virchows Arch. 2016;469:223-32.

38. Deeken-Draisey A, Yang GY, Gao J, Alexiev BA. Anaplastic thyroid carcinoma: an epidemiologic, histologic, immunohistochemical and molecular single institution study. Hum Pathol. 2018. https://doi.org/10.1016/j.humpath.2018.07.027.

39. Jeon MJ, Chun SM, Kim D, Kwon H, Jang EK, Kim TY, et al. Genomic alterations of anaplastic thyroid carcinoma detected by targeted massive parallel sequencing in a BRAF(V600E) mutation-prevalent area. Thyroid. 2016;26:683-90.

40. Oishi N, Kondo T, Ebina A, Sato Y, Akaishi J, Hino R, et al. Molecular alterations of coexisting thyroid papillary carcinoma and anaplastic carcinoma: identification of TERT mutation as an independent risk factor for transformation. Mod Pathol. 2017;30:1527-37.

41. Steck PA, Pershouse MA, Jasser SA, Yung WK, Lin H, Ligon $\mathrm{AH}$, et al. Identification of a candidate tumour suppressor gene, MMAC1, at chromosome 10q23.3 that is mutated in multiple advanced cancers. Nat Genet. 1997;15:356-62.

42. Murali R, Chandramohan R, Moller I, Scholz SL, Berger M, Huberman $\mathrm{K}$, et al. Targeted massively parallel sequencing of angiosarcomas reveals frequent activation of the mitogen activated protein kinase pathway. Oncotarget. 2015;6:36041-52.

43. Liau JY, Tsai JH, Yang CY, lee JC, Liang CW, Hsu HH, et al. Alternative lengthening of telomeres phenotype in malignant vascular tumors is highly associated with loss of ATRX expression and is frequently observed in hepatic angiosarcomas. Hum Pathol. 2015;46:1360-6. 